\title{
Exercise testing to evaluate therapeutic interventions in chronic respiratory diseases
}

\author{
Luis Puente-Maestu, MD, $\mathrm{PhD}^{1,2,3}$, William W. Stringer, MD, $\mathrm{PhD}^{4,5}$ \\ and Richard Casaburi, MD, PhD ${ }^{4,5^{\prime}}$ \\ ${ }^{1}$ Servicio de Neumología del Hospital Universitario Gregorio Marañón, Madrid, Spain; ${ }^{2}$ Instituto de investigación Sanitaria Gregorio Marañón, Madrid, Spain; \\ ${ }^{3}$ Facultad de Medicina de la Universidad Complutense de Madrid, Madrid, Spain; ${ }^{4}$ Rehabilitation Clinical Trials Center, Los Angeles BioMedical Research \\ Institute at Harbor-UCLA Medical Center, Torrance, CA, USA; ${ }^{5}$ Geffen School of Medicine at UCLA. Los Angeles, CA, USA
}

\section{ABSTRACT}

From an evidence-based perspective, performance during standardised exercise testing is of considerable importance in the multidimensional evaluation of most respiratory diseases. In this review we aim to summarise the available evidence on the responsiveness of exercise tests to various pharmacological and non-pharmacological therapeutic interventions, as well as the underlying mechanisms associated with the improvements. A standard test to evaluate interventions has not yet been defined and therefore there are several available formats with different types of responsiveness to interventions. Thresholds for clinically important changes are available for several tests. The mechanism of improvement is different depending on the disease process: when moderate or severe airflow limitation is present, as in chronic obstructive pulmonary disease (COPD), the primary mechanisms are reduced ventilatory requirement and/or enhanced ventilatory capacity via reduction of dynamic hyperinflation; in pulmonary arterial hypertension the effect of treatment is typically associated to haemodynamic improvement, while in interstitial lung diseases, the amelioration of arterial oxygen desaturation seems to be the predominant physiological factor. (BRN Rev. 2018;4(4):274-86)

Corresponding author: Luis Puente-Maestu,lpuente@separ.es

Key words: Chronic obstructive pulmonary disease. Exercise physiology. Exercise testing. Interstitial lung diseases. Pulmonary arterial hypertension.

\section{Correspondence to:}

Luis Puente-Maestu, MD, PhD

Hospital General Universitario Gregorio Marañón

Servicio de Neumología

Doctor Ezquerdo, 46

28007 Madrid, Spain

E-mail: lpuente@separ.es; lpuente.hgugm@salud.madrid.org

Received in original form: 24-03-2018

Accepted in final form: 01-05-2018

DOI: 10.23866/BRNRev:2017-0024 


\section{INTRODUCTION}

Performance during standardised exercise tests is of considerable importance in the multidimensional evaluation of most respiratory diseases $^{1}$. Exercise testing is essential for the accurate quantification of functional capacity and identifying mechanisms underlying exercise intolerance. Several physiological response indices correlate directly to major clinical outcomes, such as survival and hospital admissions, thus allowing consider able improvement in prognostic stratification ${ }^{1}$.

Recognising these important connections, regulatory agencies in the United States and Europe, such as the Food and Drug Administration (FDA) and European Medicines Agency (EMA)/Committee for Medicinal Products for Human Use (CHMP) have issued guidelines for the pharmaceutical industry acknowledging exercise testing as a primary efficacy outcome for interventions in pulmonary arterial hypertension (PAH) and a recommended complementary endpoint for chronic obstructive pulmonary disease (COPD). Direct assessment of the effects of interventions on exercise performance is likely to be relevant for other chronic respiratory conditions as well.

There is now a substantial body of evidence relating to the value of different exercise indices in assessing the effects of therapeutic interventions in respiratory diseases. The current review summarises the utility of incremental constant work rate and self-paced tests in assessing for such purpose and the physiological mechanisms underlying the responsiveness of exercise protocols to interventions.

\section{THE CONCEPT OF THE MINIMAL CLINICAL IMPORTANT DIFFERENCE}

The minimal clinically important difference (MCID) was first described in the 1980s as a method to express the smallest incremental change in a measurement that would be clinically meaningful for patients and clinicians. This differs from the concept of statistical difference, since changes in groups related to an intervention can be statistically significant (e.g., $\mathrm{p}<0.05$ ), but too small to lead to a perceptible or tangible effect on the clinical status of the patient. Commonly cited MCID values in COPD are detailed in table $1^{2}$. Minimal clinically important differences are, by nature, difficult to establish with precision, but they are usually "anchored" to the patient assessment of improvement and then triangulated by means of its relation with other measurements (changes in quality of life, survival, or COPD exacerbations among others), cut-off points derived from a specifically defined statistical distribution, such as 0.5 of the standard deviation (SD) (or 1 standard error of mean [SEM]), and the generalised clinical appraisal of relevance ${ }^{3}$. The effect seen in clinical trials is then compared with the MCID. When the mean and $95 \%$ confidence intervals (CIs) exceed the MCID, then the intervention is definitely effective (statistically and clinically significant) (Fig. 1). However, if the MCID lies within the mean and 95\% CI, then the result is statistically and clinically possible (mean result exceeds the MCID) or probably positive (result below the MCID). Finally, if the mean and 95\% CI includes the zero line on the Forest plot and is much lower than the MCID, the intervention is definitely not clinically effective (Fig. 1). 
TABLE 1. Minimal clinically important differences (MCIDs) for various important pulmonary outcome variables

\begin{tabular}{|c|c|c|}
\hline Measurement & $\begin{array}{c}\text { MCID } \\
\text { (improvement) }\end{array}$ & Method of estimation \\
\hline $\begin{array}{l}\text { Lung function } \\
\text { Trough FEV }_{1}\end{array}$ & $100 \mathrm{ml}$ & Anchor-based (exacerbations, patient perception, 2-year decline in lung function) \\
\hline Exacerbations & ?? & None \\
\hline $\begin{array}{l}\text { Dyspnoea evaluation } \\
\text { TDI } \\
\text { UCSD SOBO }\end{array}$ & $\begin{array}{l}1 \text { unit } \\
5 \text { units }\end{array}$ & $\begin{array}{l}\text { Anchor-based (physician's global evaluation score), distribution-based (SEM, } 0.5 \text { SD) } \\
\text { Anchor-based (CRO dyspnoea domain, TDI) } \\
\text { Distribution based (SEM, effect size) }\end{array}$ \\
\hline $\begin{array}{l}\text { Health status } \\
\text { SGRO }\end{array}$ & 4 units & $\begin{array}{l}\text { Anchor-based (MRC dyspnoea grade, CRO dyspnoea domain, mortality rate), expert } \\
\text { and patient preference }\end{array}$ \\
\hline $\begin{array}{l}\text { Exercise capacity } \\
\text { 6MWD } \\
\text { ISWT } \\
\text { ESWT } \\
\text { HICWR cycle }\end{array}$ & $\begin{array}{l}26 \pm 2 \mathrm{~m} \\
\text { (severe COPD) } \\
47.5 \mathrm{~m} \\
45-85 \mathrm{~s} \\
46-105 \mathrm{~s}\end{array}$ & $\begin{array}{l}\text { Anchor-based (SGRQ, UCSD SOBO), distribution based (SEM, effect size, empirical rule } \\
\text { effect size) } \\
\text { Anchor-based (patient perception) } \\
\text { Anchor-based (patient perception) } \\
\text { Distribution-based (0.5 SD) }\end{array}$ \\
\hline $\begin{array}{l}\text { Dyspnoea during exercise tests } \\
\text { Borg } \\
\text { VAS }\end{array}$ & $\begin{array}{l}1 \text { unit } \\
10-20 \text { units }\end{array}$ & $\begin{array}{l}\text { Distribution-based (effect size) } \\
\text { Distribution-based (effect size) }\end{array}$ \\
\hline
\end{tabular}

6MWD: six-minute walking distance; 6MWTD: six-minute walking distance test; COPD: chronic obstructive pulmonary disease; CR0: chronic respiratory questionnaire; ESWT: endurance shuttle walking test; FEV : forced expiratory volume in one second; HICWR: high intensity constant work rate; ISWT: incremental shuttle walking test; m: metres; ml: millilitres; MRC: Medical Research Council; SD: standard deviation; s: seconds; SEM: standard error of the mean; SGRQ: Saint George's Respiratory Questionnaire; TDI: Transition Dyspnoea Index; UCSD SOBO: University of California, San Diego Shortness Of Breath Questionnaire; VAS: visual analogic scale. (Reproduced and modified with permission from Jones PW et al. ${ }^{2}$.

\section{TEST FORMATS}

\section{Incremental exercise tests}

Incremental exercise tests (IET), including cycle or treadmill cardiopulmonary exercise tests, have the ability to report a symptomlimited measure of tolerance, i.e. peak oxygen uptake ( $\mathrm{VO}_{2}$ max) value which can be compared as a pre-post clinical intervention. This value is very reproducible (3-9\% variation) and therefore does not require more than one study to establish a baseline ${ }^{5}$. Studies in a variety of diseases, including COPD, PAH, interstitial lung diseases (ILD) and cystic fibrosis $(\mathrm{CF})^{6}$, document a strong association of
$\dot{\mathrm{VO}}_{2}$ max and survival ${ }^{7}$. Additionally, although the MCID for $\dot{\mathrm{VO}}_{2}$ max has proven difficult to ascertain, a study in COPD patients, the National Emphysema Treatment Trial (NETT), suggested that a change in $\mathrm{VO}_{2}$ max of $40 \mathrm{ml} /$ min (corresponding to a 4-watt change) was considered a MCID ${ }^{8}$. In other diseases, such as PAH, a goal of therapy is to increase the $\mathrm{VO}_{2}$ max to above $15 \mathrm{ml} / \mathrm{min} / \mathrm{kg}$ and thus improve prognosis?.

In regard to non-pharmacological therapies, studies in COPD patients with Global Initiative for Chronic Obstructive Lung Disease (GOLD) grades 2-4 have demonstrated modest increases in $\mathrm{V}_{2}$ max after lower limb 


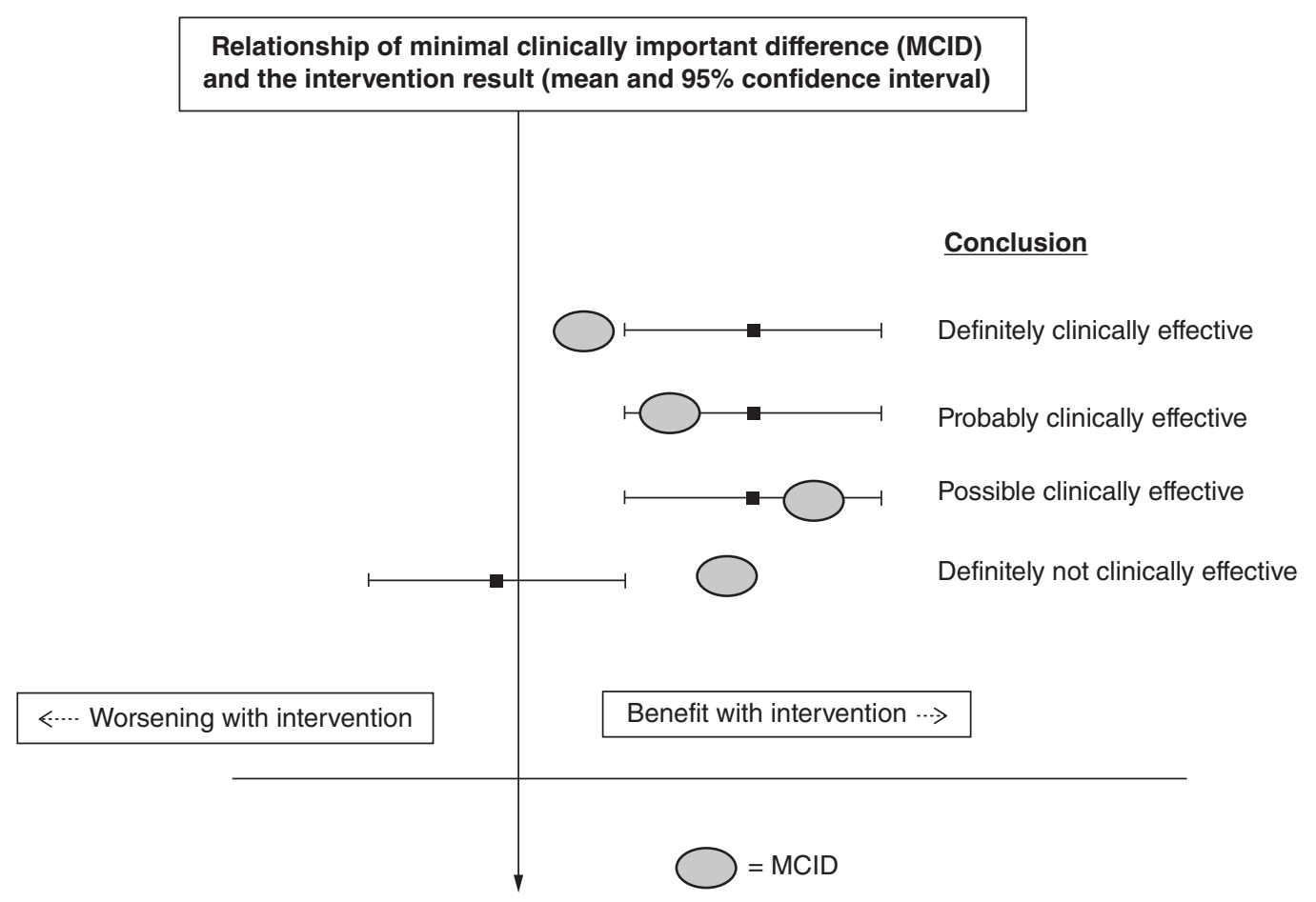

Figure 1. Relationship of minimal clinically important difference (MCID) and the intervention's result (mean and $95 \%$ confidence interval). The figure demonstrates the four types of relationships between MCID (gray circle) and the intervention mean (black square) and 95\% confidence intervals (bars). The intervention result can be larger than the MCID and the 95\% confidence intervals do not include the MCID (definitely clinically effective as an intervention). This suggests that the intervention is both statistically significant and clinically important. Probably and possibly clinically effectiveness occurs when the MCID is in the lower or upper portion of the confidence intervals, respectively. The intervention is definitely not clinically effective when the MCID is well larger than the intervention result, and the $95 \%$ confidence intervals include the null line of the Forest plot (reproduced and modified with permission from Man-Son-Hing M et al. ${ }^{4}$ ).

endurance muscle training ${ }^{10}$. Endurance training also increases $\dot{\mathrm{VO}}_{2}$ max in $\mathrm{PAH}$, in interstitial pulmonary fibrosis (IPF), in CF and in asthma ${ }^{1}$ (Table 2). The range of improvement in $\dot{\mathrm{VO}}_{2}$ max related to COPD rehabilitation is from $0.1-0.5 \mathrm{~L} / \mathrm{min}$ or about $10-40 \%$ of baseline, with a mean improvement of $11 \%$. Rehabilitation in $\mathrm{PAH}$ increases $\mathrm{VO}_{2}$ max by $1-1.5 \mathrm{ml} / \mathrm{min} / \mathrm{kg}^{11,12}$ and similarly in IPF patients ${ }^{13}$. Lung transplantation surgery, lung volume reduction surgery, supplemental oxygen therapy in COPD and in $\mathrm{CF}^{14}$ can also increase $\dot{\mathrm{VO}}_{2} \max ^{1}$.
In regard to pharmacological interventions that increase peak $\dot{\mathrm{VO}}_{2}$, short-acting $\beta_{2}$-agonist (SABA), long-acting $\beta_{2}$-agonist (LABA) and long-acting muscarinic antagonist (LAMA) bronchodilator administration can increase $\dot{\mathrm{VO}}_{2}$ max in COPD patients, although the changes are small $(40-180 \mathrm{ml} / \mathrm{min})^{1}$. Peak $\dot{\mathrm{VO}}_{2}$ has also been shown to increase with pharmacological therapy in $\mathrm{PAH}$, ranging from 1.5 to $2.0 \mathrm{ml} / \mathrm{min} / \mathrm{kg}(9-14 \% \text { change })^{15}$.

The incremental shuttle walk test (ISWT) is an externally paced evaluation ${ }^{16}$. It has been 
TABLE 2. Summary of the mechanisms involved in the improvement of exercise capacity in various pulmonary diseases

\begin{tabular}{|l|l|l|}
\hline Disease & \multicolumn{1}{|c|}{ Pharmacological interventions } & \multicolumn{1}{c}{ Endurance Training } \\
\hline $\begin{array}{c}\text { Chronic obstructive } \\
\text { pulmonary disease }\end{array}$ & $\begin{array}{l}\text { - Improved ventilatory function } \\
\text { - Improved oxygen supply to peripheral muscles }\end{array}$ & $\begin{array}{c}\text { - Improved muscle aerobic capacity (capillarisation and oxidative } \\
\text { capacity) } \\
\text { - Amelioration of dynamic hyperinflation }\end{array}$ \\
\hline $\begin{array}{c}\text { Pulmonary arterial } \\
\text { hypertension }\end{array}$ & $\begin{array}{l}\text { - Improved right ventricle function } \\
\text { - More efficient ventilation }\end{array}$ & $\begin{array}{c}\text { - Improved muscle aerobic capacity by mechanism not well character- } \\
\text { ised. Likely improvements in capillarisation and/or oxidative capacity }\end{array}$ \\
\hline $\begin{array}{c}\text { Interstitial lung } \\
\text { diseases }\end{array}$ & $\begin{array}{l}\text { - Improved arterial blood oxygenation. } \\
\text { - Amelioration of myopathic affectation }\end{array}$ & $\begin{array}{c}\text { - Improved muscle aerobic capacity by mechanism not well character- } \\
\text { ised. Likely improvements in capillarisation and/or oxidative capacity }\end{array}$ \\
\hline
\end{tabular}

applied in patient populations including COPD, ILD, and $C F^{17,18}$. The test is performed using two markers nine metres apart (ten-metre course). Walking speed is incremented each minute until the subject cannot reach the next marker in the allotted time. Small (20-25 metres) increases occur between ISWT performed on the same day and planned studies should have two ISWTs performed to establish a firm baseline, using the greater distance achieved. The MCID for ISWT has been established as 47 metres and ISWT has been shown to be a predictor of survival and readmissions in COPD patients ${ }^{17,18}$.

\section{High intensity constant work rate exercise protocols}

High intensity constant work rate (HICWR) exercise testing is commonly used to assess pharmacological and non-pharmacological interventions. These tests are typically performed on a treadmill or cycle ergometer, are preceded by an incremental test evaluation and utilise a work rate (often $70-85 \%$ of the peak work rate $[\mathrm{WR}]$ in the incremental test) sufficient to fatigue the subject within three to eight minutes. In one large series of
COPD patients, setting the intensity at $75 \%$ of peak WR resulted in values of time to tolerance $\left(t_{\text {lim }}\right)$ between three to eight minutes in $57 \%$ of the instances. When the WR was outside of the desired range, a shorter $t_{\lim }$ $(\sim 25 \%$ of tests) was more common than a longer $t_{\text {lim }}(\sim 18 \%)^{19}$. The value of $t_{\text {lim }}$ has been found to increase across two tests, five days $\operatorname{apart}^{20}$. However, average change in the second test was small (34 seconds). Other laboratories have demonstrated an even smaller average $t_{\text {lim }}$ difference in the second test on the same or next day ${ }^{21,22}$. It must be understood that, as the goal is to achieve a similar baseline $t_{1 \mathrm{lim}}$, i.e. irrespective of the subject's exercise tolerance, it would not be expected to find a relationship between baseline $t_{\lim }$ and survival, as has been demonstrated with $\dot{\mathrm{VO}}_{2}$ max and ISWT peaks. Therefore, HICWR protocols are only of use for judging the effectiveness of interventional protocols. The MCID for change in $t_{\text {lim }}$ is approximately 105 seconds. Results of $t_{\text {lim }}$ have been shown to be responsive to both pharmacological (e.g., bronchodilators) and non-pharmacological interventions (e.g., pulmonary rehabilitation). For bronchodilators, changes in $t_{\lim }$ approximating the MCID have been reported, whereas pulmonary 
rehabilitation showed increases in $t_{\lim }$ well exceeding the MCID.

The endurance shuttle walk testing (ESWT) is designed from the results of the ISWT; after a 100-second warm up period at a slow pace, the recommended speed (often $80 \%$ of the peak ISWT) is instituted. Results can be expressed in feet or metres.

The available evidence suggests that, in COPD, endurance tests are more responsive to interventions, both pharmacological and non-pharmacological, than incremental tests (i.e., IET and ISWT) and the six-minute walking distance test $(6 \mathrm{MWD})^{1}$.

\section{Self-paced exercise tests}

The $6 \mathrm{MWD}$ is an example of a self-paced test. The subject walks back and forth on an indoor 30-metre flat corridor for six minutes and the distance or laps are recorded ${ }^{17}$. As there is a learning effect, the largest (feet or metres) of the two tests should be used as baseline to any therapeutic intervention ${ }^{18}$. The MCID is felt to be between 25-33 meters regardless of the disease state. Six-minute walking distance $(6 \mathrm{MWD})$ can stratify mortality risk in $\mathrm{COPD}^{23}$ and is part of the Body mass index, airflow Obstruction, Dyspnoea, and Exercise capacity (BODE) index ${ }^{24}$. The 6MWD is often used to assess the physiological improvement with pulmonary rehabilitation. According to a recent Cochrane Review of 38 trials, the mean effect of pulmonary rehabilitation on 6MWD is 44 metres $(95 \%$ CI 33 to 55$)^{25}$. In patients with PAH the $6 \mathrm{MWD}$ keeps good correlation with improvements in haemodynamics ${ }^{26}$, however it does not appear to be an optimal marker of changes in relevant clinical outcomes such as the time to clinical deterioration or survival ${ }^{27}$. Exercise training in IPF showed an improvement in 6MWD of 39 metres $^{28}$. A limitation of the 6MWD test is its low responsiveness to bronchodilator interventions in COPD, with many studies reporting mean results that are below the MCID value (SABA, 20-42 metres, SAMA, 6-39 metres, and LABA, 21-54 metres) ${ }^{1,29}$.

\section{FACTORS DETERMINING CHANGES IN EXERCISE OUTCOMES AFTER INTERVENTIONS}

\section{Dynamic hyperinflation}

Since dynamic hyperinflation (DH) is one of the major determinants of exercise capacity and symptoms in patients with expiratory airflow limitation ${ }^{30-33}$, the relevance of interventions on its time course during exercise is relevant for the following discussion.

The primary effect of bronchodilator therapy is to increase the resting inspiratory capacity while the rate of $\mathrm{DH}$ during exercise is generally not decreased ${ }^{32,34}$. Therefore, bronchodilators delay the restriction on tidal volume imposed by the hyperinflation of the lungs, allowing a greater minute ventilation ${ }^{32,34}$ and, by reducing the operating lung volume, effectively improve inspiratory muscle performance $^{32,34}$ and lessen dyspnoea ${ }^{32}$. Additionally, amelioration of DH results in a host of secondary effects with potentially additive beneficial effects on exercise capacity. Thus, $\mathrm{DH}$ diminution improves the lung-heart mechanical interaction, improving cardiac function ${ }^{35,36}$ and lowers the oxygen cost of 
breathing $^{32}$, freeing up blood flow from the respiratory muscles to be delivered to the periphery ${ }^{37}$.

Leg muscle training in COPD patients has also been shown to reduce $\mathrm{DH}^{1}$. The mechanisms appear to involve a reduction in lactic acid production by the exercising muscle yielding reduced ventilatory requirements, such that $\dot{V}_{\mathrm{E}}$ and respiratory rate are lower at isotime ${ }^{1}$. Consequently, patients with airflow limitation can exercise for longer because the point at which a critical level of dynamic hyperinflation is reached is delayed.

\section{Ventilatory efficiency}

The ventilatory equivalent for carbon dioxide $\left(\dot{\mathrm{V}}_{\mathrm{E}}-\dot{\mathrm{V} C \mathrm{O}_{2}}\right)$ (i.e., the slope between $\dot{\mathrm{V}}_{\mathrm{E}}$ and $\dot{\mathrm{V} C O} \mathrm{O}_{2}$ or the minimal respiratory equivalent of $\mathrm{CO}_{2}$ during incremental exercise tests or the respiratory equivalent at the lactate threshold) are increased in most respiratory and cardiac diseases reflecting increased pulmonary dead space $\left(\mathrm{V}_{\mathrm{D}} / \mathrm{V}_{\mathrm{T}}\right)$ or decreased arterial partial pressure of carbon dioxide $\left(\mathrm{PaCO}_{2}\right)$ or both ${ }^{38}$. In PAH, while both increased $\mathrm{V}_{\mathrm{D}} / \mathrm{V}_{\mathrm{T}}$ and reduced $\mathrm{PaCO}_{2}$ play a role, $\mathrm{V}_{\mathrm{D}} / \mathrm{V}_{\mathrm{T}}$ is typically the main determining factor ${ }^{15}$. Moreover, in these patients, $\dot{\mathrm{V}}_{\mathrm{E}}-\dot{\mathrm{V}} \mathrm{CO}_{2}$ significantly correlates with decreased cardiac output and particularly with increased pulmonary vascular resistance $(\mathrm{PVR})^{15}$. The ventilatory equivalent for carbon dioxide $\left(\dot{\mathrm{V}}_{\mathrm{E}}-\mathrm{CO}_{2}\right)$ is also increased in chronic respiratory patients ${ }^{39}$ mainly as a result of increased $\mathrm{V}_{\mathrm{D}} / \mathrm{V}_{\mathrm{T}}$ because of ventilation-perfusion mismatching ${ }^{40,41}$, the adoption of an inefficient breathing patter $n^{40}$ or concomitant heart failure ${ }^{42}$. More efficient ventilation has been shown after pulmonary rehabilitation in $\mathrm{COPD}^{43,44}$ and bronchodilator therapy ${ }^{45}$, which will likely result in reduced $\dot{\mathrm{V}}_{\mathrm{E}}-\dot{\mathrm{VCO}}_{2}$.

\section{Maximal aerobic capacity}

The locus of limitation in maximal oxygen flow could occur at any point between the lungs and the mitochondria of exercising muscles. In patients with COPD with low forced expiratory volume in one second $\left(\mathrm{FEV}_{1}\right)^{30}$, decreased peak $\mathrm{VO}_{2}$ is typically dictated by (a) an elevated ventilatory requirement related both to increased $\mathrm{V}_{\mathrm{D}} / \mathrm{V}_{\mathrm{T}}{ }^{31}$, and also to premature metabolic acidosis due to muscle deconditioning/dysfunction ${ }^{10,31}$ and in some cases arterial hypoxaemia ${ }^{31}$, and (b) lowered ventilatory capacity ${ }^{31,46}$ and increased dyspnoea at a given level of $\dot{V}_{\mathrm{E}^{\prime}}$ in which the increased respiratory impedance due to $\mathrm{DH}$ plays a major role $30-32,41,47$. The increases in peak $\mathrm{VO}_{2}$ seen after training in COPD patients usually reflect a decreased ventilatory requirement ${ }^{43,44}$. A minority of these patients have significant pulmonary arterial hypertension and in those, low cardiac output may limit exercise performance (Table 2$)^{48}$.

While patients with primary PAH show extremely inefficient ventilation (with high $\left.\mathrm{V}_{\mathrm{D}} / \mathrm{V}_{\mathrm{T}}\right)^{49}$, which may in some cases be the limiting factor for exercise, poor right cardiac function and arterial hypoxaemia are the main causes of low aerobic capacity ${ }^{15,50}$. Therefore, $\dot{\mathrm{VO}}_{2}$ max improvements are usually a consequence of reduced pulmonary artery pressure and improved cardiac output ${ }^{51,52}$. As in other chronic respiratory diseases, $\mathrm{PAH}$ results in muscle deconditioning/dysfunction which likely play a role in exercise limitation, 
since endurance training, increases $\mathrm{VO}_{2}$ max in patients with PAH (Table 2) $)^{11,12}$.

In regard to patients with ILD, respiratory mechanical abnormalities appear not to be the predominant cause for exercise limitation in the majority of patients ${ }^{40,53}$. It is likely that the marked arterial oxygen desaturation, which is a common feature of ILD during exercise, plays a significant role, especially in patients with $\mathrm{IPF}^{54}$, since correction of arterial hypoxaemia improves $\dot{\mathrm{V}}_{2}{ }^{55}$ max (Table 2). While ventilation-perfusion mismatching is the primary contributor to the arterial hypoxaemia at rest and during exercise in ILD, diffusion limitation to oxygen transfer, due to a short transit time of the red blood cells through the pulmonary capillaries, also plays an important role ${ }^{56}$. This has been observed in patients with ILD of various aetiologies; nonetheless, there are differences in the extent of the gas exchange disturbance during exercise, which differ somewhat according to diagnosis. On average, patients with IPF show a larger increase in the alveolar-arterial partial pressure of oxygen difference $\left(\mathrm{AaPO}_{2}\right)$ during exercise than those with sarcoidosis ${ }^{57}$ or asbestosis ${ }^{58}$. It has been hypothesised that these differences are related to the degree of underlying pulmonary interstitial fibrosis, as exercise-induced oxygen arterial desaturation correlates with the degree and distribution of parenchymal abnormalities on lung imaging ${ }^{59}$. Circulatory limitation can also determine maximal exercise performance in some patients with $\mathrm{ILD}^{53}$. The type of circulatory limitation depends on the disease state. For example, pulmonary hypertension is not uncommon in patients with IPF, with a strong association between elevation in pulmonary artery pressure and reduction in $\mathrm{VO}_{2} \max ^{60}$, and it has been reported to be the primary limitation to exercise in patients with systemic sclerosis and ILD ${ }^{61}$. However, current treatments for pulmonary hypertension in patients with IPF have not shown a significant effect on exercise intolerance ${ }^{55}$. Cardiac dysfunction has been documented during exercise in patients with sarcoidosis, even in the absence of overt signs of cardiac involvement, lung function impairment or arterial oxygen desaturation ${ }^{62}$. This may represent subclinical granulomatous infiltration of the myocardium that can be treated with glucocorticoids ${ }^{62}$.

Peripheral muscle dysfunction can be another contributor to exercise limitation in ILD. Thus, quadriceps muscle force is an independent predictor of peak $\mathrm{VO}_{2}$ both in sarcoidosis ${ }^{63}$ and $\mathrm{IPF}^{28}$. It is likely that a component of the muscle dysfunction is related to deconditioning, since it responds to training (Table 2) ${ }^{28}$. However, chronic oral glucocorticoid administration may contribute in some patients ${ }^{63}$.

\section{Endurance}

Endurance is typically measured by constant WR testing. In healthy subjects ${ }^{64}$, patients with $\mathrm{COPD}^{65}$, and likely in other respiratory diseases (i.e. pulmonary hypertension, interstitial pulmonary diseases, cystic fibrosis, etc.), the relationship between WR and $t_{\text {lim }}$ for constant-WR high intensity exercise is hyperbolic (Fig. 2) according to the equation,

$$
\mathrm{t}_{\lim }=\mathrm{W}^{\prime} /(\mathrm{WR}-\mathrm{CP})^{1}
$$

where $W^{\prime}$ is the curvature constant. Critical power $(\mathrm{CP})$ represents the highest $\mathrm{WR}$ that can be maintained without $\mathrm{VO}_{2}$ continuing to increase with time to $\mathrm{VO}_{2}$ max rather than 


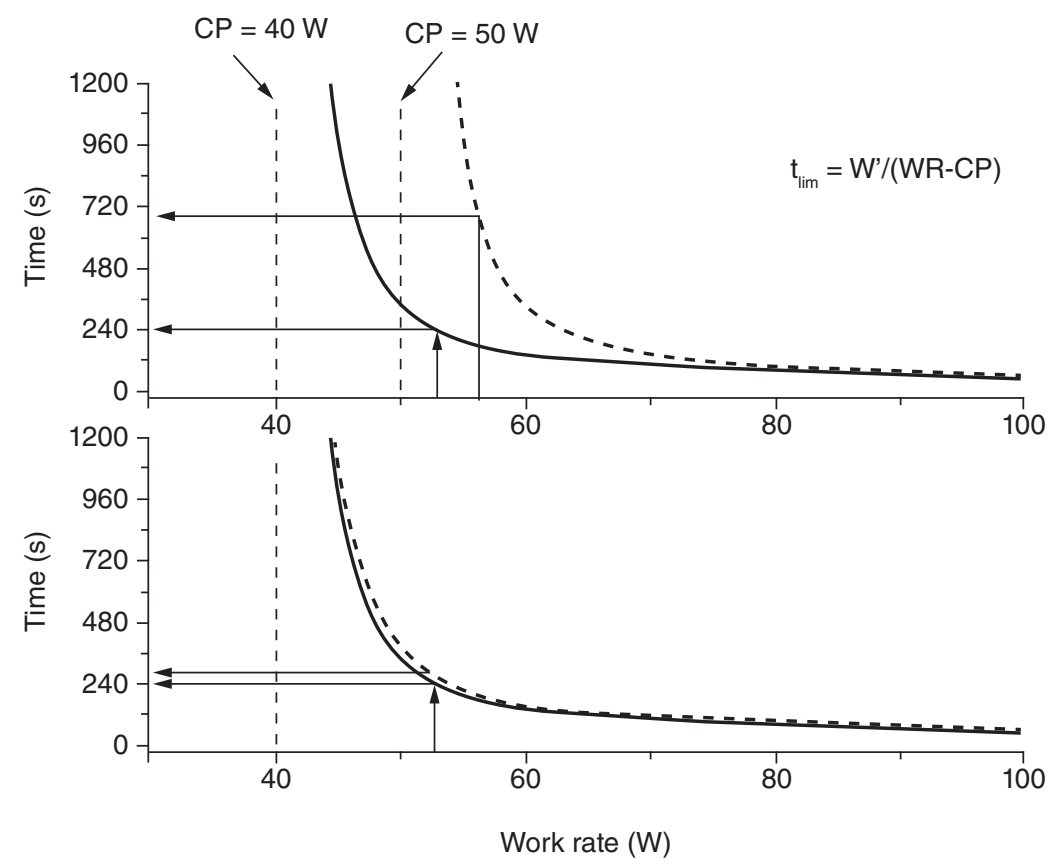

Figure 2. Effects of changes in critical power or curvature on endurance time of high intensity exercise.

The figure depicts the effects of time of changes on critical power (CP) from 40 to $50 \mathrm{~W}$ (upper panel) and curvature constant (lower panel) from 3000 to $3500 \mathrm{~J}$ on the power-duration relationship, resulting in a given high intensity work rate being sustainable for a longer period. Solid line denotes pre-intervention; dashed line denotes post-intervention.

6MWD: six-minute walking distance test; CP: critical power; $t_{\text {lim }}$ : limit of endurance; $W^{\prime}$ : curvature constant; WR: work rate.

attaining submaximal steady-state conditions. Furthermore, in normal subjects, arterial $\mathrm{pH}$ can stabilise up to CP but not at higher work rates $^{64,65}$. It has also been described as the fatigue threshold or the limits of aerobic equilibrium during exercise ${ }^{64}$.

In healthy subjects, $\mathrm{W}^{\prime}$ is felt to reflect a finite energy store comprised of intramuscular high-energy phosphates (adenosine triphosphate [ATP], phosphocreatine), glycogen and stored oxygen, whose rate of depletion contributes to the establishment of $\mathrm{t}_{\lim }{ }^{64}$. Alternatively, $t_{\lim }$ may be determined by the rate at which fatigue-related processes (i.e. intramuscular accumulation of inorganic phosphate, potassium or $\mathrm{H}^{+}$ions) accumulate to some critical tolerable limit ${ }^{64}$. Endurance time may also be dictated by the tolerance to the uncomfortable sensations that arise when the systems involved are approaching to their limit ${ }^{65}$.

From the hyperbolic shape of the power-duration relationship (Fig. 2), it can be inferred that the impact of interventions on $t_{\lim }$ will depend on a complex interplay between $\mathrm{CP}$, $\mathrm{W}^{\prime}$ and the WR selected for the test ${ }^{64,65}$. Both $\mathrm{CP}$ and $\mathrm{W}^{\prime}$ can increase in COPD after endurance training ${ }^{65}$, the latter because of improved respiratory mechanical factors and/or (perhaps) dyspnoea desensitisation. Minor improvements 
in $\mathrm{CP}$ have a profound improvement in endurance $t_{\text {lim }}$ if the WR is adequately selected. This is likely the reason for the greater responsiveness of this kind of tests to most interventions compared with other tests ${ }^{1}$. There is no direct evidence on the effects of bronchodilator therapy on the power-duration relationship in COPD patients. However, as bronchodilators are known to increase the maximal voluntary ventilation and decrease $\mathrm{DH}^{1}, \mathrm{~W}^{\prime}$ might be increased as a result. Furthermore, unloading respiratory muscles by bronchodilator therapy increases locomotor muscle blood flow ${ }^{37}$ and -by reducing $\mathrm{DH}$ - improves cardiac function as well ${ }^{35,36}$. Both phenomena can increase CP. Endurance time also improves in hypoxaemic patients with supplemental oxygen ${ }^{66}$, mostly due to a slowing of the breathing pattern that forestalls the point at which dynamic hyperinflation results in ventilatory limitation, so that $\mathrm{CP}$ is thereby increased ${ }^{1}$. Other interventions aimed at unloading the respiratory system, such as breathing a helium/oxygen mixture, noninvasive ventilatory support or lung volume reduction surgery, may affect endurance time by similar mechanisms ${ }^{1}$.

After vasodilator therapy endurance improvements in patients with primary $\mathrm{PAH}$ correlates with changes in cardiac index and pulmonary vascular resistance ${ }^{67}$ and the likely associated increase in CP.

\section{Six-minute walking distance test}

During the 6MWD, the patient is encouraged to cover as much distance as possible in six minutes. Patients select the speed at which they perform the test and seem to do so in order to be able to walk at a sustainable speed. Therefore, although the 6MWD is not intended to be a constant WR test, several studies have shown that chronic respiratory patients tend to choose a relatively constant speed of walking, and hence $\mathrm{WR}^{68,69}$, as shown by the attainment of a plateau in the $\dot{\mathrm{VO}}_{2}$ max and heart rate responses during the test $\mathrm{t}^{1,68,69}$. Moreover, on average, COPD patients have been reported to select a walking speed during the 6MWD that is below the critical speed (the equivalent of $\mathrm{CP}$ for walking $)^{70}$. The $6 \mathrm{MWD}$ has two particular features that make it different and potentially less sensitive to interventions than other exercise tests ${ }^{1}$. First, an improved 6MWD requires an increased walking speed, as has been reported in patients with COPD after locomotor muscle training (which included treadmill walking) ${ }^{71}$. Less readily accommodated, however, is the observation that COPD patients adopted similar walking speeds for 6MWD performed, following a placebo and a bronchodilator ${ }^{1,72}$, suggesting that patients may tend to reproduce the same walking speed during repeated 6MWDs, irrespective of bronchodilator administration. This could explain why the $6 \mathrm{MWT}$ is an unresponsive exercise test for evaluating the effects of bronchodilator therapy. Second, the 6MWD test has a "ceiling" effect (Fig. 3) as the linear relationship between $\dot{\mathrm{VO}}_{2}$ max and 6MWD is lost in less impaired subjects since they are closer to their maximal walking speed ${ }^{1,73}$. In patients with primary PAH, 6MWD increases are correlated with improved haemodynamic, particularly with improved PVR ${ }^{1,27}$.

The pooled analysis of the studies of pirfenidone in IPF demonstrated that after 72 weeks of treatment with this medication there was 


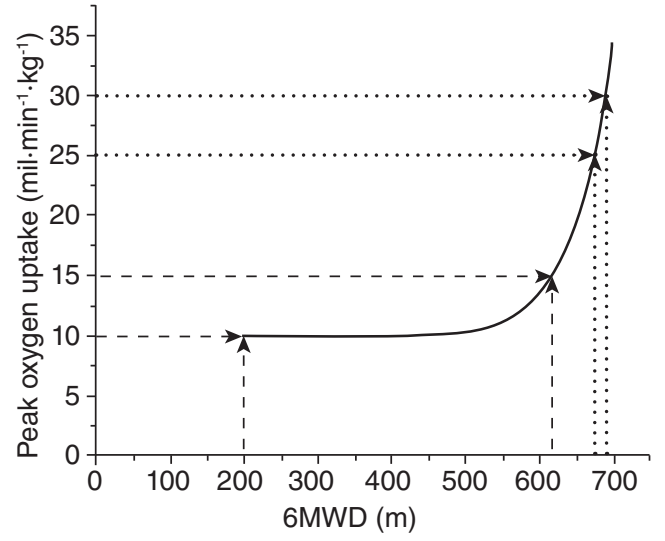

Figure 3. "Ceiling" effect for the six-minute walking test (6MWD). This figure graphically shows the "ceiling" effect for the 6MWD. In less sick patients (i.e. $>20 \mathrm{ml} \cdot \mathrm{min}^{-1} \cdot \mathrm{kg}^{-1}$ ) similar improvements in aerobic capacity ( $\mathrm{Y}$ axis) (dotted line) are translated into a smaller increase in the distance walked ( $X$ axis) than in patients with a lower baseline aerobic capacity (dashed line) (i.e. $<20 \mathrm{ml} \cdot \mathrm{min}^{-1} \cdot \mathrm{kg}^{-1}$ ). The relationship between aerobic capacity and 6MWD was modelled based on reference 73 . 6MWD: six-minute walking distance test.

a statistically significant difference in 6MWD between the treatment and control groups of the order of 24 metres $^{74}$. The mechanism for improvement in endurance after the anti-fibrotic treatment remains uncertain, since both lung function and gas exchange deteriorated in those studies ${ }^{74}$.

\section{CONCLUSIONS}

We have attempted to summarise the available evidence on the responsiveness of exercise tests to various pharmacological and non-pharmacological therapeutic interventions, as well as the underlying mechanisms associated with the achieved improvements.
As yet, a "gold standard" test has not been identified, and the choice of testing modality is both disease- and intervention-specific.

In addition, the mechanism of improvement from the intervention is different depending on the disease process. In COPD, it focuses on $\mathrm{DH}$, while in pulmonary hypertension the focus is on improvements in cardiac function and in patients with interstitial lung disease on both gas exchange and hypoxia as well as cardiac function.

\section{ACKNOWLEDGEMENTS}

To Professor Karlman Wasserman and Professor Brian Whipp pioneers of exercise physiology and admired scientist and teachers.

\section{CONFLICTS OF INTEREST}

Authors have nothing to disclose in relation to the submitted work.

\section{REFERENCES}

1. Puente-Maestu L, Palange P, Casaburi R et al. Use of exercise testing in the evaluation of interventional efficacy: an official ERS statement. Eur Respir J. 2016;47:429-60.

2. Jones PW, Beeh KM, Chapman KR, Decramer M, Mahler DA, Wedzicha JA. Minimal clinically important differences in pharmacological trials. Am J Respir Crit Care Med. 2014;189:250-5.

3. Norman GR, Sloan JA, Wyrwich KW. Interpretation of changes in health-related quality of life: the remarkable universality of half a standard deviation. Med Care. 2003;41:582-92.

4. Man-Son-Hing M, Laupacis A, O'Rourke K et al. Determination of the clinical importance of study results. J Gen Intern Med. 2002;17:469-76.

5. ATS/ACCP Statement on cardiopulmonary exercise testing. Am J Respir Crit Care Med. 2003;167:211-77.

6. Pianosi P, Leblanc J, Almudevar A. Peak oxygen uptake and mortality in children with cystic fibrosis. Thorax. 2005;60:50-4.

7. Palange P, Ward SA, Carlsen KH et al. Recommendations on the use of exercise testing in clinical practice. Eur Respir J. 2007;29:185-209.

8. Puhan MA, Chandra D, Mosenifar Z et al. The minimal important difference of exercise tests in severe COPD. Eur Respir J. 2011;37:784-90. 
9. McLaughlin VV, Gaine SP, Howard LS et al. Treatment goals of pulmonary hypertension. J Am Coll Cardiol. 2013;62(Suppl):D73-81.

10. Casaburi R, Patessio A, Ioli F, Zanaboni S, Donner CF, Wasserman K. Reductions in exercise lactic acidosis and ventilation as a result of exercise training in patients with obstructive lung disease. Am Rev Respir Dis. 1991;143:9-18

11. Grunig E, Lichtblau M, Ehlken N et al. Safety and efficacy of exercise training in various forms of pulmonary hypertension. Eur Respir J. 2012;40:84-92.

12. Zafrir B. Exercise training and rehabilitation in pulmonary arterial hypertension: rationale and current data evaluation. J Cardiopulm Rehabil Prev. 2013;33:263-73.

13. Dowman L, Hill CJ, Holland AE. Pulmonary rehabilitation for interstitial lung disease. Cochrane Database Syst Rev. 2014:CD006322.

14. Elphick HE, Mallory G. Oxygen therapy for cystic fibrosis. Cochrane Database Syst Rev. 2013:CD003884.

15. Arena R, Lavie CJ, Milani RV, Myers J, Guazzi M. Cardiopulmonary exercise testing in patients with pulmonary arterial hypertension: an evidence-based review. J Heart Lung Transplant. 2010;29:159-73.

16. Singh SJ, Morgan MD, Scott S, Walters D, Hardman AE. Development of a shuttle walking test of disability in patients with chronic airways obstruction. Thorax. 1992;47:1019-24.

17. Holland AE, Spruit MA, Troosters T et al. An official European Respiratory Society/American Thoracic Society technical standard: field walking tests in chronic respiratory disease. Eur Respir J. 2014;44:1428-46.

18. Singh SJ, Puhan MA, Andrianopoulos V et al. An official systematic review of the European Respiratory Society/American Thoracic Society: measurement properties of field walking tests in chronic respiratory disease. Eur Respir J. 2014;44:1447-78.

19. Andrianopoulos V, Wagers SS, Groenen MT et al. Characteristics and determinants of endurance cycle ergometry and six-minute walk distance in patients with COPD. BMC Pulm Med. 2014;14:97.

20. O'Donnell DE, Travers J, Webb KA et al. Reliability of ventilatory parameters during cycle ergometry in multicentre trials in COPD. Eur Respir J. 2009;34:866-74

21. van 't Hul A, Gosselink R, Kwakkel G. Constant-load cycle endurance performance: test-retest reliability and validity in patients with COPD. J Cardiopulm Rehabil. 2003;23:143-50.

22. Puente-Maestu L, Villar F, de Miguel J et al. Clinical relevance of constant power exercise duration changes in COPD. Eur Respir J. 2009;34:340-5.

23. Cote CG, Pinto-Plata V, Kasprzyk K, Dordelly LJ, Celli BR. The 6-min walk distance, peak oxygen uptake, and mortality in COPD. Chest. 2007;132: 1778-85.

24. Celli BR, Cote CG, Marin JM, Casanova C et al. The body-mass index, airflow obstruction, dyspnea, and exercise capacity index in chronic obstructive pulmonary disease. N Engl J Med. 2004;350:1005-12.

25. McCarthy B, Casey D, Devane D, Murphy K, Murphy E, Lacasse Y. Pulmonary rehabilitation for chronic obstructive pulmonary disease. Cochrane Database Syst Rev. 2015:CD003793.

26. Gabler NB, French B, Strom BL et al. Validation of 6-minute walk distance as a surrogate end point in pulmonary arterial hypertension trials. Circulation. 2012;126:349-56.

27. Macchia A, Marchioli R, Tognoni G et al. Systematic review of trials using vasodilators in pulmonary arterial hypertension: why a new approach is needed. Am Heart J. 2010;159:245-57.

28. Holland A, Hill C. Physical training for interstitial lung disease. Cochrane Database Syst Rev. 2008:CD006322.

29. Borel B, Provencher S, Saey D, Maltais F. Responsiveness of Various Exercise-Testing Protocols to Therapeutic Interventions in COPD. Pulm Med. 2013;2013:410748

30. Casaburi R, Maltais F, Porszasz J et al. Effects of tiotropium on hyperinflation and treadmill exercise tolerance in mild to moderate chronic obstructive pulmonary disease. Ann Am Thorac Soc. 2014;11:1351-61.

31. Gallagher CG. Exercise limitation and clinical exercise testing in chronic obstructive pulmonary disease. Clin Chest Med. 1994;15:305-26.
32. O'Donnell DE, Hamilton AL, Webb KA. Sensory-mechanical relationships during high-intensity, constant-work-rate exercise in COPD. J Appl Physiol. 2006;101:1025-35.

33. Puente-Maestu L, Garcia de PJ, Martinez-Abad Y, Ruiz de Ona JM, Llorente D, Cubillo JM. Dyspnea, ventilatory pattern, and changes in dynamic hyperinflation related to the intensity of constant work rate exercise in COPD. Chest. 2005;128:651-6.

34. O'Donnell DE, Revill SM, Webb KA. Dynamic hyperinflation and exercise intolerance in chronic obstructive pulmonary disease. Am J Respir Crit Care Med. 2001;164:770-7.

35. Hohlfeld JM, Vogel-Claussen J, Biller $\mathrm{H}$ et al. Effect of lung deflation with indacaterol plus glycopyrronium on ventricular filling in patients with hyperinflation and COPD (CLAIM): a double-blind, randomised, crossover, placebo-controlled, single-centre trial. Lancet Respir Med. 2018;6 368-78.

36. Vassaux C, Torre-Bouscoulet L, Zeineldine $\mathrm{S}$ et al. Effects of hyperinflation on the oxygen pulse as a marker of cardiac performance in COPD. Eur Respir J. 2008;32:1275-82.

37. Berton DC, Barbosa PB, Takara LS et al. Bronchodilators accelerate the dynamics of muscle $\mathrm{O} 2$ delivery and utilisation during exercise in COPD. Thorax. 2010;65:588-93.

38. Wasserman K, Hansen JE, Sue DY, Stringer WW, Sietsema KE, Sun XG Principles of Exercise Testing and Interpretation: Including Pathophysiology and Clinical Applications. Fifth ed. Philadelphia: Lippincott Williams \& Wilkins. 2011.

39. Sun XG, Hansen JE, Garatachea N, Storer TW, Wasserman K. Ventilatory efficiency during exercise in healthy subjects. Am J Respir Crit Care Med. 2002;166:1443-8.

40. Marciniuk DD, Gallagher CG. Clinical exercise testing in interstitial lung disease. Clin Chest Med. 1994;15:287-303.

41. Gallagher CG. Exercise limitation and clinical exercise testing in chronic obstructive pulmonary disease. Clin Chest Med. 1994;15:305-26.

42. Rocha A, Arbex FF, Sperandio PA et al. Excess Ventilation in Chronic Obstructive Pulmonary Disease-Heart Failure Overlap. Implications for Dyspnea and Exercise Intolerance. Am J Respir Crit Care Med. 2017;196:1264-74.

43. Puente-Maestu L, Sanz ML, Sanz P, Ruiz de Ona JM, Rodriguez-Hermosa JL, Whipp BJ. Effects of two types of training on pulmonary and cardiac responses to moderate exercise in patients with COPD. Eur Respir J. 2000 15:1026-32.

44. Troosters T, Casaburi R, Gosselink R, Decramer M. Pulmonary rehabilitation in chronic obstructive pulmonary disease. Am J Respir Crit Care Med. 2005;172:19-38.

45. O'Donnell DE, Voduc N, Fitzpatrick M, Webb KA. Effect of salmeterol on the ventilatory response to exercise in chronic obstructive pulmonary disease. Eur Respir J. 2004;24:86-94.

46. Neder JA, Jones PW, Nery LE, Whipp BJ. Determinants of the exercise endurance capacity in patients with chronic obstructive pulmonary disease The power-duration relationship. Am J Respir Crit Care Med. 2000;162 497-504.

47. Puente-Maestu L, Garcia de Pedro J, Martinez-Abad Y, Ruiz de Ona JM, Llorente D, Cubillo JM. Dyspnea, ventilatory pattern, and changes in dynamic hyperinflation related to the intensity of constant work rate exercise in COPD. Chest. 2005;128:651-6.

48. Seeger W, Adir Y, Barbera JA et al. Pulmonary hypertension in chronic lung diseases. J Am Coll Cardiol. 2013;62:D109-D16.

49. Deboeck G, Niset G, Lamotte M, Vachiery JL, Naeije R. Exercise testing in pulmonary arterial hypertension and in chronic heart failure. Eur Respir J. 2004;23:747-51

50. Sun XG, Hansen JE, Oudiz RJ, Wasserman K. Exercise pathophysiology in patients with primary pulmonary hypertension. Circulation. 2001;104: 429-35.

51. Oudiz RJ, Roveran G, Hansen JE, Sun XG, Wasserman K. Effect of sildenafil on ventilatory efficiency and exercise tolerance in pulmonary hypertension. Eur J Heart Fail. 2007;9:917-21. 
52. Barst RJ, Langleben D, Frost A et al. Sitaxsentan therapy for pulmonary arterial hypertension. Am J Respir Crit Care Med. 2004;169:441-7.

53. Hansen JE, Wasserman K. Pathophysiology of activity limitation in patients with interstitial lung disease. Chest. 1996;109:1566-76.

54. Miki K, Maekura R, Hiraga T et al. Impairments and prognostic factors for survival in patients with idiopathic pulmonary fibrosis. Respir Med. 2003; 97:482-90.

55. Bajwah S, Ross JR, Peacock JL et al. Interventions to improve symptoms and quality of life of patients with fibrotic interstitial lung disease: a systematic review of the literature. Thorax. 2013;68:867-79.

56. Agusti AG, Roca J, Gea J, Wagner PD, Xaubet A, Rodriguez-Roisin R. Mechanisms of gas-exchange impairment in idiopathic pulmonary fibrosis. Am Rev Respir Dis. 1991;143:219-25.

57. Risk C, Epler GR, Gaensler EA. Exercise alveolar-arterial oxygen pressure difference in interstitial lung disease. Chest. 1984;85:69-74.

58. Agusti AG, Roca J, Rodriguez-Roisin R, Xaubet A, Agusti-Vidal A. Different patterns of gas exchange response to exercise in asbestosis and idiopathic pulmonary fibrosis. Eur Respir J. 1988;1:510-6.

59. Sette A, Neder JA, Nery LE et al. Thin-section CT abnormalities and pulmonary gas exchange impairment in workers exposed to asbestos. Radiology. 2004;232:66-74.

60. Gläser S, Noga O, Koch B et al. Impact of pulmonary hypertension on gas exchange and exercise capacity in patients with pulmonary fibrosis. Respir Med. 2009;103:317-24.

61. Sudduth CD, Strange C, Cook WR et al. Failure of the circulatory system limits exercise performance in patients with systemic sclerosis. Am J Med. 1993;95:413-8

62. Gibbons WJ, Levy RD, Nava S et al. Subclinical cardiac dysfunction in sarcoidosis. Chest. 1991;100:44-50.

63. Spruit MA, Thomeer MJ, Gosselink R et al. Skeletal muscle weakness in patients with sarcoidosis and its relationship with exercise intolerance and reduced health status. Thorax. 2005;60:32-8.
64. Poole DC, Burnley M, Vanhatalo A, Rossiter HB, Jones AM. Critical Power: An Important Fatigue Threshold in Exercise Physiology. Med Sci Sports Exerc. 2016;48:2320-34

65. Puente-Maestu L, SantaCruz A, Vargas T, Martinez-Abad Y, Whipp BJ Effects of training on the tolerance to high-intensity exercise in patients with severe COPD. Respiration. 2003;70:367-70.

66. Bradley JM, Lasserson T, Elborn S, Macmahon J, O'neill B. A systematic review of randomized controlled trials examining the short-term benefit of ambulatory oxygen in COPD. Chest. 2007;131:278-85.

67. Savarese G, Musella F, D'Amore C et al. Haemodynamics, exercise capacity and clinical events in pulmonary arterial hypertension. Eur Respir J. 2013;42:414-24.

68. Troosters T, Vilaro J, Capit n A, Rabinovich RA, Vilar E, Gonzalez C, et al Organ system responses during the six minute walking test. Am J Respir Crit Care Med. 2001;163:A268.

69. Casas A, Vilaro J, Rabinovich R, Mayer A, et al. Encouraged 6-min walking test indicates maximum sustainable exercise in COPD patients. Chest. 2005 128:55-61.

70. Troosters T, Vilaro J, Rabinovich R et al. Physiological responses to the 6-min walk test in patients with chronic obstructive pulmonary disease. Eur Respir J. 2002;20:564-9.

71. Dolmage TE, Evans RA, Hill K, Blouin M, Brooks D, Goldstein RS. The effect of pulmonary rehabilitation on critical walk speed in patients with COPD: a comparison with self-paced walks. Chest. 2012;141:413-9.

72. Laviolette L, Bourbeau J, Bernard S et al. Assessing the impact of pulmonary rehabilitation on functional status in COPD. Thorax. 2008;63:115-21.

73. Lipkin DP, Scriven AJ, Crake T, Poole-Wilson PA. Six minute walking test for assessing exercise capacity in chronic heart failure. Br Med J (Clin Res Ed). 1986;292:653-5.

74. Noble PW, Albera C, Bradford WZ et al. Pirfenidone in patients with idiopathic pulmonary fibrosis (CAPACITY): two randomised trials. Lancet. 2011;377:1760-9. 